\section{VULNERABILIDAD ANIMAL EN REFUGIOS ESPAÑOLES: SITUACIONES DE EMERGENCIA Y DESASTRE. FILOMENA TAMBIÉN ES NOMBRE DE ANIMAL}

\author{
Eduardo Barona Collado ${ }^{1 *}$ y Pedro Tomé Martín ${ }^{2}$
}

\section{RESUMEN}

Los desastres no afectan a todos por igual. La vulnerabilidad de cada individuo se construye social y culturalmente. Si conceptos como clase social, edad o etnicidad resultan importantes, pertenecer a una determinada especie es determinante ante un desastre. Este trabajo aborda el concepto de vulnerabilidad de los animales residentes en refugios españoles, en situaciones de emergencia y desastre. Para ello reconstruye etnográficamente las historias de 4 refugios de animales durante el paso de la borrasca "Filomena" por España, a principios de 2021. El artículo enfatiza la necesidad de incluir a los no humanos en la narrativa humanitaria, así como en el desarrollo de tecnologías, como leyes, protocolos de actuación y planes de respuesta, que garanticen su seguridad, promoviendo una responsabilidad tanto de la administración pública como de las entidades privadas. A partir del estudio de caso de la borrasca Filomena en España, este trabajo pretende evidenciar la vulnerabilidad de los animales de compañía residentes en refugios y protectoras, abriendo una vía para su incorporación en la gestión de emergencias y desastres de cualquier índole, que resulte aplicable a todas las regiones del planeta, con especial foco en Latino América, Caribe y España.

\section{PALABRAS CLAVES}

Estudios Humano-Animal, Humanitarismo, Vulnerabilidad, Animales en desastres

ANIMAL VULNERABILITY IN SPANISH SHELTERS: EMERGENCY AND DISASTER SITUATIONS. FILOMENA IS ALSO THE NAME OF AN ANIMAL

\section{ABSTRACT}

Disasters do not affect everyone equally. Vulnerability of each individual is constructed socially and culturally. If concepts such as social class, age or ethnicity are important, belonging to a certain species is decisive when the disaster strikes. This work addresses the concept of vulnerability of animals residing in Spanish shelters, in emergency and disaster situations. To do this, it ethnographically reconstructs the stories of 4 animal shelters during the storm "Filomena" over Spain, at the beginning of 2021. The article emphasizes the need to include non-humans in the humanitarian narrative, as well as in the development of technologies, such as laws, action protocols and response plans, which guarantee their safety, promoting a responsibility of both the public administration and the private entities. Based on the case study of the storm Filomena in Spain, this paper aims to show the vulnerability of companion animals residing in shelters and animal NGO's, opening a way for their incorporation in the management of emergencies and disasters of any kind, which is applicable to all regions of the planet, with a special focus on Latin America, the Caribbean and Spain.

\author{
1. Universidad Complutense de \\ Madrid, Madrid, España. \\ 2. Consejo Superior de \\ Investigaciones Científicas \\ (CSIC), Madrid, España. \\ *Autor de correspondencia: \\ ebaronac@ucm.es
}

DOI:

https://doi.org/10.55467/ reder.v6i1.86

\section{RECIBIDO}

16 de septiembre de 2021

\section{ACEPTADO}

12 de diciembre de 2021

\section{PUBLICADO}

1 de enero de 2022

\section{Formato cita}

Recomendada (APA):

Barona Collado, E. \&

Tomé Martín, P. (2022)

Vulnerabilidad animal en refugios españoles: Situaciones de emergencia y desastre. Filomena también es nombre de animal. Revista de Estudios Latinoamericanos sobre Reducción del Riesgo de Desastres REDER, 6(1), 99111. https://doi.org/10.55467/ reder.v6i1.86

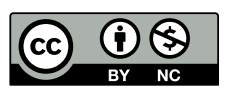

Todos los artículos publicados en REDER siguen una política de Acceso Abierto y se respaldan en una Licencia CreativeCommons Atribución-NoComercial 4.0 Internacional.

Revista de Estudios Latinoamericanos sobre Reducción del Riesgo de Desastres (REDER)

Diseño: Lupe Bezzina

\title{
KEYWORDS
}

Human-Animal Studies, Humanitarianism, Vulnerability, Animals in disasters 


\section{INTRODUCCIÓN}

En "Filling the ark. Animal welfare in disarters", Irvine (2009) plantea un dilema ético enmarcado en el discurso moderno sobre el humanitarismo. Sugiere la autora que solo cuando un riesgo se materializa, se produce una emergencia y que sólo cuando la emergencia supera la capacidad de respuesta, sobreviene el desastre. Atendiendo a esta idea, los riesgos se pueden clasificar por su origen en naturales 0 antropogénicos. Sin embargo, los desastres se construyen a partir de categorías socialmente establecidas, es decir no hay "desastres naturales", sino que son el resultado de las condiciones de vulnerabilidad a las que se exponen los individuos (Oliver-Smith, 2009). Entonces, si las cuestiones de raza, género o clase social han sido contempladas como factores que influyen en la vulnerabilidad de los individuos en situaciones de desastre, ¿por qué no incluir también la pertenencia a una determinada especie como un factor más? Es más, ¿qué especies animales merecerian, en esa situación, nuestra consideración moral y cuáles no?, Si llega el diluvio, ¿a quién salvamos primero? y ¿a quién dejamos fuera de nuestra arca?.

Miriam Ticktin $(2014,2015)$ lleva años trabajando para tensar los límites del humanitarismo, contribuyendo con una propuesta expansiva más allá de lo humano, que contemple a los demás animales y en general a todo el planeta. Bajo esta mirada, el humanitarismo es el sentimiento humano de querer aliviar el sufrimiento de "los otros", pero no debe limitarse al humano como beneficiario. Barnett (2011) vino a definir el humanitarismo como una "revolución de la ética del cuidado", una responsabilidad hacia el bienestar ajeno, que alcanza una significación aún mayor, en el llamado Antropoceno, periodo en el que nuestras actividades impactan significativamente sobre los demás animales y el planeta (De Paula \& Anthony, 2021). Sin embargo, esta ética de reverencia por la vida, no puede quedarse solo en el plano de las ideas, sino que se debe articular también en un sentido pragmático (Tobias \& Morrison, 2017).

En este segundo ámbito, la propia Ticktin (2014) observa la necesidad de desplegar discursos, así como prácticas, infraestructuras, procedimientos, leyes, entre otros. Todas estas tecnologías aplicadas deben ser incorporadas a la narrativa humanitaria, contribuyendo a la inclusión de los no humanos de una manera interdependiente, esto es, con una contribución performativa al concepto del humanitarismo. Una de las áreas en las que el humanitarismo descansa más claramente en el desarrollo de estas tecnologías es el manejo de emergencias y desastres, en el que se busca aliviar el sufrimiento provocado por estas situaciones (Ticktin, 2015). Sin embargo, este ámbito de estudio, que está ampliamente desarrollado en EEUU, en referencia a la gestión de desastres provocados reiteradamente por el paso de huracanes, no tiene apenas representación en otras regiones del planeta, pese a la clara evidencia de que el cambio climático está incrementando los fenómenos climatológicos extremos a nivel global. En Latino América, dada su alta exposición a determinados fenómenos, también se vienen haciendo importantes contribuciones a esta área, tanto desde el sector agropecuario, como en lo que se refiere a animales de compañía (Morales et al., 2017; Hernández-Pulido et al., 2021; Díaz et al., 2015; Yglesias, 2015; Alfonso \& Percedo, 2020).

Este trabajo aborda la necesidad de incluir a los animales no humanos en nuestras preocupaciones cuando sobrevienen situaciones de emergencia y desastre, bien sean de carácter "natural" o antropogénico. En él, se pretende desplegar tecnologías, en forma de prácticas, procedimientos y leyes que sean capaces de mediar en la gestión de emergencias y desastres que afectan a los animales no humanos, tomando como base cuatro ejemplos etnográficos a partir de las experiencias de diferentes refugios de animales, durante la emergencia humanitaria provocada por el paso de la borrasca "Filomena" en la península Ibérica entre el 6 y el 11 de enero de 2021.

Aunque existen algunos trabajos sobre la gestión de los animales en situaciones de emergencia y desastre, éstos se han venido aplicando mayoritariamente a la capacidad de respuesta del sector agropecuario, en particular para animales de gran volumen y peso (Gimenez et al., 2002; Howlett \& Turnbull, 2009). Mientras que la mayoría de los que mencionaron expresamente a los animales de compañía, lo hicieron por su valor logístico y emocional para las personas. A diferencia de ellos, este trabajo pretende una incorporación de los animales de compañía en la gestión de emergencias y catástrofes, no basada en criterios de bienestar humano. De este modo es, hasta nuestro conocimiento, el primero en su clase, tanto en Latino América como en Europa. 


\section{METODOLOGÍA}

El texto inicia con una revisión bibliográfica de la literatura académica sobre la atención a animales no humanos en situaciones de emergencia, nutriéndose fundamentalmente de la experiencia de EEUU en este tipo de fenómenos. A continuación, se describen cuatro viñetas etnográficas, basadas en testimonios de diferentes voluntarios de organizaciones de protección de los animales, introduciendo el caso concreto de los refugios españoles. El tercer apartado se centra en la descripción del concepto de vulnerabilidad, así como de las singularidades aplicables al contexto de los refugios españoles. En el cuarto apartado se expone la necesidad de desplegar diferentes tecnologías, como leyes, prácticas y procedimientos estandarizados, así como de formación sobre planes de respuesta y evacuación en emergencias y desastres, que puedan afectar a refugios y centros que alberguen animales. Finalmente, en las conclusiones se resumen algunos resultados de esta investigación.

El objetivo de este trabajo es indagar en la vulnerabilidad de determinados animales que residen en refugios para animales "de compañía" en el momento en que se dan situaciones de emergencia y desastre. Los autores, somos conscientes de que los desastres no afectan solo a estos animales, sino también a otras poblaciones de animales no humanos que, de hecho, suelen permanecen invisibles en estas situaciones, como los empleados para la alimentación, la investigación o trabajo, o los utilizados en circos, zoos, espectáculos ambulantes o competiciones, legales o ilegales, así como los animales silvestres que viven en libertad (Dennison \& Lin, 2013). No los incluimos aquí pues nos centramos en la vulnerabilidad singular de determinados colectivos de animales, así como en la necesidad de desarrollar tecnologías para aliviar su sufrimiento, dentro de un marco que pretende avanzar en su consideración moral.

\section{ANIMALES EN EMERGENCIAS Y DESASTRES}

Existe una extensa bibliografía sobre los animales "de compañía" en desastres, especialmente en EEUU y lo que se refiere al paso de huracanes. Sin embargo, muchos de estos trabajos tienen un marcado sesgo antropocéntrico, al centrarse más en cómo la compañía de un animal influye en las decisiones que los humanos deben tomar, que en el incremento de vulnerabilidad de los animales que dependen de ellos (Hunt et al., 2012; Petrolia \& Bhattacharjee, 2010). La mayoría de estos trabajos muestran cómo las personas que tienen animales no humanos bajo su responsabilidad, son más reacias a abandonar sus casas en situaciones de emergencia, no solo para no dejar a sus animales atrás, sino también por temor a no poder acudir a un lugar seguro en compañía de su animal (Travers et al., 2017; Heath et al., 2001; Buttke et al., 2013). Pese a ser enfoques centrados en las decisiones humanas, estos trabajos ponen en evidencia los profundos vínculos que unen a los humanos con los demás animales que están bajo su responsabilidad y, por lo tanto, la importancia de contemplar a los animales "de compañía" en los planes de emergencia estatales y locales (Zottarelli, 2010).

La producción académica sobre esta temática ha venido centrándose en el desarrollo de planes de evacuación y gestión de emergencias, que integren también a los animales "de compañía" (Glassey \& Wilson, 2011), la necesidad de establecer refugios pet-friendly para que las personas puedan ser trasladadas junto con sus animales, disminuyendo así la vulnerabilidad de ambos (Douglas et al., 2017; Farmer et al., 2016; Farmer \& DeYoung, 2019) o la importancia de desarrollar planes de evacuación y gestión de emergencias, tanto de nivel estatal, como a nivel local y particular (Farmer et al., 2016).

En EEUU, la Stafford Act, otorga a la Agencia Federal para el Manejo de Emergencias (FEMA) la coordinación de los planes de actuación ante situaciones de emergencia y desastre. Esta agencia provee un mecanismo estandarizado para el manejo de los incidentes, conocido como Incident Command System (ICS) que se organiza en cuatro niveles: Táctico, financiero, de planificación y logístico (Dennison \& Lin, 2013). En el caso de EEUU, los avances en la consideración moral de los animales "de compañía", sumados a una mayor visibilización a través de los medios, se vieron acentuados por la difícil situación vivida durante el paso del Huracán Katrina en 2005, para terminar, impulsando en 2006, el desarrollo de la "Pet Evacuation and Transportation Standards Act". Esta herramienta legislativa, conocida como PETS Act, es coordinada por la FEMA a través del modelo ICS, instando a incluir a los animales "de compañía" en los planes de respuesta a emergencias (Irvine, 2009; Dennison \& Lin, 2013; Madigan \& Dacre, 2009). 
Sin embargo, esta iniciativa que integra la medicina veterinaria en la gestión de emergencias, no implica tecnologías y procedimientos específicos, por lo que necesita ser reforzada en la práctica por el trabajo de campo de numerosas entidades de carácter público y privado o mixto, tanto a nivel local como estatal. Cabe citar, entre otros, el Veterinary Medical Assistance Team (VMAT), el Disaster Animal Rescue Team (DART), los programas Community Animal Response Team (CART) y State Animal Response Team (SART), así como diferentes grupos profesionales de trabajo coordinados por la National Alliance of State Animal and Agricultural Emergency Programs (NASAAEP) (Dieckmann et al., 2020).

Más allá de todos estos avances, poca atención se ha centrado en la posibilidad de que el desastre sobrevenga en un refugio o centro de recuperación y/o adopción de animales. En una revisión bibliográfica que realizaron Travers, Degeling y Rock (2017), solo dos trabajos fueron hallados sobre este caso. En el primero de ellos, Irvine (2007), narra la situación vivida durante un simulacro de emergencia y evacuación llevado a cabo en un refugio urbano de animales. La simulación de una emergencia provocada por un escape de gas, permitió testar la capacidad de respuesta del centro y refinar su modelo operacional, estableciendo sus necesidades logísticas y de formación. En el segundo trabajo (Decker et al., 2010), se realizaron encuestas a 115 refugios de animales, en el estado de Ohio (EEUU), pudiendo confirmar algunas de las observaciones de Irvine. La primera de ellas, que existe una falta de preparación ante situaciones de emergencia y desastre por parte del voluntariado y staff. Irvine (2007) definió este perfil de voluntariado como "bien intencionado", aunque escasamente formado. La segunda conclusión fue, que, aunque la mayoría de los encuestados valoraron la importancia de desarrollar planes de respuesta, solo el $13 \%$ disponían de alguno.

Como se verá, la vulnerabilidad de los animales albergados en este tipo de centros en España, podría ser muy superior a la de los centros estadounidenses. Dado el carácter recurrente de los incidentes locales, así como la predicción de un incremento de riesgos de diverso tipo, sería oportuno llamar la atención sobre la necesidad de impulsar leyes y procedimientos estatales y autonómicos, así como planes de emergencia que contemplen la planificación, preparación, mitigación, respuesta y recuperación (Heath \& Linnabary, 2015).

\section{TECNOLOGÍAS PARA UN HUMANITARISMO EXPANSIVO}

Propone Ticktin $(2014,2015)$ el desarrollo de tecnologías que deben contribuir a un ensanchamiento del concepto de humanitarismo que de cabida no solo a los humanos, sino también a los demás animales, así como a la globalidad de nuestro planeta. Sin embargo, el despliegue de estas tecnologías no solo ha de venir a disminuir las vulnerabilidades expuestas, sino también a formar parte de un discurso que obedece a la lógica expansiva del humanitarismo. Observa la autora, cómo en el contexto de las emergencias y desastres, este nuevo humanitarismo se basa en el alivio del sufrimiento, operando a través de infraestructuras, técnicas, kits, protocolos, así como de herramientas legislativas y de defensa de la integridad física de los individuos. Todas estas tecnologías tienen cabida en la Gestión del Riesgo de Desastres (GRD, o DRM, de sus siglas en inglés). En lo que refiere a la gestión de los animales no humanos, Heath \& Linnabary (2015), establecen 5 fases: planificación, preparación, mitigación, respuesta y recuperación, a las que nos vamos a referir brevemente.

La más amplia de todas, la primera de ellas, llamada fase de planificación, trata de identificar y priorizar las amenazas, riesgos y vulnerabilidades a las que se expone cada comunidad 0 , en nuestro caso, cada refugio, definiendo misiones y objetivos a cumplir en caso de que acontezcan. En esta fase se desarrollan los diferentes planes de respuesta y protocolos que contemplen a los animales no humanos en situaciones de emergencia. Con el objetivo de estandarizar guías operacionales que eviten subjetividades e interpretaciones en situaciones de emergencia y desastre. Si en el modelo estadounidense son los diferentes estados los que se encargan de bajar al nivel micro esta norma a través de protocolos y planes específicos (Farmer et al., 2016), en España, sería previsiblemente, la Dirección General de Protección Civil y Emergencias, así como la Unidad Militar de Emergencias, las responsables de trabajar coordinadamente con la Dirección General de los Derechos de los Animales (DGDDAA), en la elaboración herramientas legislativas de carácter estatal, así como de la elaboración de protocolos y procedimientos integrados dentro de un modelo ICS (Dennison \& Lin, 2013; Madigan \& Dacre, 2009). Esta acción, se debe completar con la formación y coordinación de equipos de respuesta estatales (SART's) o comunitarios (CART's). 
La Dirección General de los Derechos de los Animales, dependiente de la Secretaría de Estado para la Agenda 2030, pertenece al Ministerio de Derechos Sociales y Agenda 2030. A través del artículo 10 del Real Decreto 452/2020, de 10 de marzo', se establecen sus funciones, que abarcan todo lo relativo a la formulación de políticas en materia de protección de los derechos de los animales, así como la coordinación con el resto de las administraciones públicas y privadas en materia de derechos de los animales y su protección.

Además, cada entidad debe también elaborar planes de respuesta y recuperación propios, por escrito, así como protocolos de evacuación que contemplen el alojamiento y las necesidades básicas de los animales albergados, incluidas las posibles complejidades, así como los tratamientos veterinarios necesarios. Para este nivel, se establece la formación de Equipos de Rescate de Animales en Desastres (DART's). La existencia de distintos niveles de intervención requiere de un esfuerzo notable en la coordinación y movilización para la respuesta, que incluye también las relaciones entre entidades para posibles acuerdos de colaboración.

La segunda fase, de las propuestas por Heath \& Linnabary (2015), fase de preparación, hace referencia a la certificación y preparación de todos estos equipos veterinarios, así como de intervención y rescate, que serán los primeros respondedores en caso de emergencia. En EEUU, FEMA se encarga de desarrollar estos cursos de entrenamiento y formación, así como de la acreditación de los asistentes y de la accesibilidad financiera a los mismos, garantizando así la existencia de un voluntariado cualificado (Irvine, 2004, 2009). En España, la responsable de la coordinación sería la DGDDAA junto con la Dirección General de Protección Civil y Emergencias y la Unidad Militar de Emergencias.

La tercera fase, de mitigación, se define como la alteración permanente del medio para minimizar los riesgos ante un desastre. La adecuación de las instalaciones y refuerzo de los accesos y rutas de transporte ante eventuales fenómenos "naturales" o antropogénicos, como inundaciones, incendios, rachas de viento, terremotos... se contemplan en esta fase. Se requiere, por lo tanto, de dotaciones económicas adecuadas, así como, una vez más, del trabajo coordinado de las diferentes agencias.

La cuarta fase, la de respuesta, tiene que ver con la discordancia temporal que existe entre la necesidad de proteger las vidas y los recursos disponibles para ello. Es el momento en el que las condiciones preexistentes, que definen la vulnerabilidad, se exacerban y transformarán totalmente la última fase, la de recuperación, que solo se podrá iniciar tras el desastre, aunque puede demorarse en el tiempo. En todo caso, el trabajo previo de identificación de las vulnerabilidades, que debe realizarse en la planificación, tendrá también un impacto directo en la capacidad de respuesta. Así se deduce de los testimonios de varios voluntarios cuyos refugios se vieron afectados por el paso de la borrasca Filomena, a principios de 2021.

\section{RESULTADOS. 4 VIÑETAS ETNOGRÁFICAS DURANTE EL PASO DE FILOMENA}

La borrasca nombrada por AEMET como "Filomena" fue la sexta de la temporada 2020-2021, dejando un temporal de viento y lluvias en toda la península lbérica, entre el 6 y el 10 de enero de 2021, así como nevadas copiosas en amplias zonas del interior. La histórica nevada caída durante los días 8 y 9 de enero, dio paso a una ola de frío que se alargó hasta el 17 de enero, formando placas de hielo que acarrearon múltiples problemas en las comunicaciones, servicios y suministros (AEMET, 2021).

\section{Cuencanimal}

La protectora Cuencanimal cuenta con un refugio propio en una zona rural, a unos 15 kilómetros de Cuenca, en el que, desde 2011 albergan una población cercana a los 100 perros. Se trata de una entidad local donde las personas responsables mantienen relaciones cercanas. El episodio del paso de Filomena por su refugio fue un golpe al que vino a sumarse la muerte de Mercedes Culebras, su presidenta y fundadora, solo un mes y medio más tarde.

Dice Purificación García, vicepresidenta e íntima amiga de Mercedes, que la presidenta era ese tipo de persona con un don de gentes excepcional. Esa suerte de diplomacia les ha venido permitiendo mantener unas buenas relaciones tanto con entidades de protección animal en Francia, que les apoyan en las adopciones internacionales de perros españoles, así como también con las instituciones públicas locales. Gracias a estas relaciones se dio difusión de su caso en televisión, así como en redes sociales, recibiendo bastantes donaciones particulares para paliar
1. Real Decreto 452/2020, de 10 de marzo, por el que se desarrolla la estructura orgánica básica del Ministerio de Derechos Sociales Agenda 2030, y se modifica el Real Decreto $139 / 2020$, de 28 de enero, por el que se establece la estructura orgánica básica de los departamentos ministeriales. marzo de 2020, páginas 24611 a 24625 . Referencia: BOE-A-2020-3512. 
los problemas a los que se enfrentaron. Para Purificación, "Puri", la buena reputación de la entidad fue la que evitó males mayores durante el paso del temporal.

Más allá de algunos daños materiales como boxes hundidos por el peso de la nieve y los problemas con la congelación del agua, tanto para beber como para limpiar, el principal problema con que se encontraron fue el acceso desde la carretera principal. Aunque pronto un Ayuntamiento cercano mandó un vehículo pick-up con pala y surtidora de sal, el camino rural, quedó intransitable al tráfico durante más de una semana. Por fortuna, Filomena les pilló con un pedido de comida deshidratada para perros recién llegado al refugio, por lo que en ningún momento peligró su alimentación. Sin embargo, el paso del temporal si les ha hecho valorar la creación de un plan de emergencias ante posibles catástrofes como incendios, dada la ubicación del refugio en una zona boscosa. Como en otros casos, la posibilidad de que un gran número de animales sin capacidad de huida, se vean sorprendidos por una emergencia, los hace extremadamente vulnerables.

\section{APAP-Alcalá}

La protectora San Martín de Porres es propietaria de los terrenos donde construyó su refugio, a unos $50 \mathrm{~km}$ de Madrid. Actualmente viven en él unos 80 perros separados en módulos y perreras para evitar peleas. El acceso se realiza a través de un camino de tierra de $300 \mathrm{~m}$ que separa este albergue de la carretera asfaltada más próxima. Por motivos de seguridad, se establecen turnos entre los trabajadores para pernoctar en el centro. Según el testimonio de Lourdes, coordinadora del voluntariado en la entidad, la noche que comenzó la nevada, Elena, trabajadora del refugio, se encontraba cubriendo este turno. El camino quedó completamente bloqueado durante los dos 0 tres días siguientes, dejando a Elena aislada hasta que otros dos trabajadores, Sergio y Fernando, pudieron acceder a pie tras varias horas de camino, para darle el relevo y compartir la responsabilidad de la atención a los perros. Durante este periodo no recibieron apoyo o asistencia de protección civil ni de ninguna entidad pública, teniendo que ser autosuficientes en la gestión de la emergencia.

Aunque mantuvieron el contacto por WhatsApp en todo momento y no dejaron de apoyarla, para Elena fueron días muy duros, pues la acumulación de nieve impedía la apertura de la puerta de los módulos para la limpieza y alimentación de los perros, teniendo que lanzar la comida desde arriba de las vallas. Además, la congelación del agua impedía tanto la limpieza como la hidratación de estos animales, obligándoles a la ingesta de nieve con el consiguiente riesgo de hemorragia digestiva. Por fortuna, más allá de algunos suelos dañados, que deberán sustituir, no han tenido mayores daños materiales y no hubo que lamentar la muerte de ningún animal.

Sin embargo, Lourdes enfatiza en que el refugio de APAP-Alcalá, como muchos otros, se encuentra en un lugar alejado y poco accesible, donde los precios de compra de los terrenos son asequibles y los ayuntamientos conceden las licencias para establecer núcleos zoológicos, pero también donde la vulnerabilidad de los animales ante situaciones de emergencia se eleva drásticamente. En su caso, como consecuencia de la experiencia vivida, se movilizaron para conseguir una ayuda del Ayuntamiento más próximo, para cubrir con zahorra el camino y así mejorar el acceso, aunque ésta, finalmente no ha llegado. Por otro lado, lo sucedido, les ha servido para reflexionar sobre la necesidad de establecer protocolos de emergencias.

\section{Brinzal}

El caso de Brinzal es diferente a los anteriores. Patricia Orejas, coordinadora de esta ONG que trabaja para la recuperación de rapaces nocturnas, es consciente de sus múltiples singularidades. En primer lugar, porque son un centro de recuperación privado, que trabaja con fauna silvestre y que opera en terrenos cedidos por la Comunidad de Madrid, dentro del albergue juvenil de la Casa de Campo (Madrid). En segundo lugar, porque el objetivo que se persigue es la recuperación y liberación de rapaces heridas, enfermas o huérfanas, entregadas por ciudadanos particulares, así como agentes forestales o policía local. Trabajar con este tipo de animales implica entender, que, para ellos, los humanos somos depredadores y no sus benefactores. Estas dos condiciones hacen que las instalaciones de Brinzal estén especialmente orientadas a evitar la huida de las aves y esta característica es clave para contemplar su vulnerabilidad.

Para las aves heridas, enfermas o huérfanas se dispone de una zona de hospitalización, que, afortunadamente, se encontraba vacía durante los días del paso de Filomena. Para las rapaces en su última fase de recuperación se dispone de voladeros, que les permiten aprender a volar y recuperar musculación, previamente a su liberación. Se trata de estancias grandes construidas 
con postes de madera y mallas metálicas donde las rapaces disponen de espacio para iniciar sus vuelos. Por las características de estas instalaciones, el peso de la nieve acumulada ocasionó el hundimiento casi total de 8 de estas infraestructuras, donde murieron una lechuza y un cárabo y de donde se escapó otra ave. También en la zona nodriza, que alberga animales irrecuperables para su vida en libertad, cayó un poste que permitió escapar a un búho chico, que finalmente pudo ser recuperado, dada su escasa movilidad.

La noche del viernes 8 de enero no había nadie en el centro. Durante todo el sábado el acceso fue imposible. Finalmente, el domingo 10, Patricia, junto con otros voluntarios, pudieron llegar a pie recorriendo con gran dificultad el kilómetro y medio que separa las instalaciones de la parada de metro más próxima. Afortunadamente suelen guardar un día de ayuno para las aves, para evitar la acumulación excesiva de grasa, pudiendo hacerlo coincidir con el sábado que no pudieron acceder. En ese momento, unos 80 animales se encontraban en el centro, 26 de ellos en las instalaciones que resultaron dañadas.

Patricia reconoce con tristeza que en solo unas horas se echaron a perder 35 años de trabajo para conseguir unas infraestructuras óptimas. Sin embargo, comenta que en ningún momento se sintieron abandonados. El lunes 11 recibió la llamada de diferentes administraciones públicas, ofreciendo su colaboración. Del mismo modo, reconoce que, siendo vistos como una entidad de colaboración con la ciudadanía, hubo una respuesta magnífica, tanto en la elaboración de grupos de voluntariado como en la recaudación de fondos para la reconstrucción. Su observación muestra como la ayuda no se dirigió tanto a las aves en sí, sino más bien hacia las necesidades de la ONG y voluntariado, cuyo objetivo si es el del bienestar de las rapaces. De igual forma, otras entidades de protección animal se sumaron a estas ayudas, estableciendo redes de solidaridad que les han permitido continuar con su actividad.

\section{AiBA Madrid}

AiBA Madrid es una entidad privada constituida en 2012, que dispone de un refugio propio para gatos y colabora con el CPA (Centro de Protección Animal) de Valdemoro (Madrid), prestando soporte para la gestión de los animales sin responsable (perros y gatos) en la localidad y brindando una red de casas de acogida para los mismos. Actualmente alberga a 20 perros en el CPA y 40 gatos en su local. Además de ello se encargan de la gestión sanitaria y esterilización de la población felina en Valdemoro, así como de la organización de su voluntariado.

Laura Martínez narra cómo, si bien su local no estuvo afectado por el paso del temporal, pues varias voluntarias viven cerca y pudieron acceder pese a las dificultades, en esos días recibieron numerosos avisos de ciudadanos en general y de los voluntarios que gestionan las colonias de gatos, en particular, por sus dificultades de acceso. Muchas personas se encontraron con la dificultad de acceder a los gatos, a los que escuchaban maullar. Estos avisos se pasaron a la empresa privada Fomento de Construcciones y Contratas (FCC), que es quién tiene la concesión por parte del Ayuntamiento de Valdemoro, para la gestión del CPA. Ellos mismos abrieron las vías con tractores y quitanieves para acceder al CPA. Las infraestructuras de este centro fueron financiadas con fondos públicos y resistieron bien la nevada. Dada la situación de bloqueo, Laura se plantea las enormes dificultades que hubiera supuesto la necesidad de ayuda de la administración pública en esos días.

El caso de AiBA Madrid, ilustra la situación vivida por numerosas personas dedicadas al voluntariado de alimentación y gestión de natalidad de las colonias urbanas de gatos. A diferencia de los casos anteriores, la vulnerabilidad de los gatos, no se debe a su limitación de movimiento sino a su condición de "callejeros" y su dependencia alimentaria.

\section{DISCUSIÓN. VULNERABILIDAD DE LOS ANIMALES RESIDENTES EN REFUGIOS ESPAÑOLES}

Reconocer las formas en las que los humanos explotamos el medio ambiente para sustento y refugio propio, así como de los demás animales, es clave en el estudio de la vulnerabilidad y evolución de los desastres (Oliver-Smith, 2009). Esto implica contemplar nuestras relaciones con el medio desde una perspectiva que integre los diferentes planos multiespecie, lo que nos llevaría a una aproximación diferenciada que contempla la vulnerabilidad animal dentro de una política ecológica de los desastres (Oliver-Smith, 2009). 
Como se ha mencionado, los desastres e incidentes locales, vienen a poner de manifiesto las vulnerabilidades previas de una determinada comunidad, exacerbando las condiciones preexistentes (Heath \& Linnabary, 2015). Terremotos, lluvias torrenciales que derivan en inundaciones, nevadas, olas de calor, incendios, escapes tóxicos, entre otros, no son problemáticos en sí, sino que la vulnerabilidad y la exposición de los humanos y demás animales a estos eventos extremos, son los que determinan la materialización y el riesgo de desastre. En esas situaciones, cuestiones como la clase social, el género, la edad, la etnicidad, la religión, así como las propias capacidades corporales del individuo, se han contemplado como factores capaces de incrementar o disminuir la vulnerabilidad (Bankoff, 2004). Irvine (2009) añade también la pertenencia a una u otra especie como un factor más. Para ello, alude a la llamada "Escala sociozoológica" propuesta por Arluke y Sanders (1996), que transmite la idea de que nuestras interacciones con los demás animales están condicionadas por el lugar que les otorgamos a través de una categorización jerárquica.

En esa escala, los Ilamados animales "de compañia", de los que se encarga mayoritariamente este artículo, ocupan un lugar privilegiado frente a otros, debido principalmente a una mayor visbililización y vínculo emocional con los humanos (Farmer et al., 2016). Consecuentemente, la mayor parte de la bibliografía existente sobre animales en emergencias y desastres, se basa en la gestión de las "mascotas" en este tipo de situaciones. Sin embargo, es necesario decir que su condición de "domésticos" también los torna totalmente dependientes de la consideración humana, aumentando así su vulnerabilidad (Irvine, 2007; Mattes, 2018; Bankoff, 2014). Es decir, la paradoja de los animales "de compañía" radica en que, pese a estar situados en lo alto de esta escala sociozoológica, podrían verse expuestos a una alta vulnerabilidad social por su total dependencia de los humanos (Vieira \& Anthony, 2021). Esta dependencia, que implica la responsabilidad hacia el cuidado de los animales, incluso en situaciones extremas, es claramente expuesto en la situación vivida por Elena, en el ejemplo etnográfico de APAP-Alcalá y constituye un área emergente en este ámbito, pues contribuye a la comprensión de las intrincadas relaciones entre humanos y demás animales (Travers et al., 2017).

En todo caso, se ha venido prestando poca atención a aquellos lugares destinados a la recuperación y/o adopción de animales (domésticos o silvestres), donde múltiples factores se relacionan con un incremento de la vulnerabilidad ante un riesgo. Algunos de ellos, como el elevado número de animales concentrados, la precariedad de las infraestructuras y su ubicación espacial, el estado de salud de los animales residentes, la escasa formación del voluntariado, la invisibilidad para la administración pública o la falta de consideración moral de una parte de la población hacia estos animales, podrían sobre-expresar esta vulnerabilidad en el caso de los refugios españoles.

El número de animales residentes en refugios españoles, viene manteniendo, según la Fundación Affinity, un incremento cercano al 16\% anual (Fatjó \& Calvo, 2018). En las encuestas llevadas a cabo por Decker et al. (2010), sobre 115 agencias de cuidado animal, en el estado de Ohio, se comunicó una media de 29 perreras y 5 gateras. Si bien no existen datos fiables del conjunto del país, aunque sí de algunas localidades, los existentes ponen de manifiesto que, en España, el número de abandonos de perros es superior a los que hay en el resto de países de su entorno. Si a ello se le añade que el número de identificaciones y esterilizaciones es menor, resulta altamente probable que el número de cánidos que precisan ser recogidos en algún refugio sea muy elevado. Considerando la baja tasa de adopciones de animales residentes en refugios, cabe suponer que, en la mayor parte de los casos, el número de animales residentes, puede exceder de aquel para el que las infraestructuras están adaptadas y diseñadas. De hecho, en todos los ejemplos mencionados en este artículo, se superaban ampliamente estas cifras.

Por un lado, la dificultad de establecer un número concreto de animales en una determinada área, constituye una enorme dificultad a la hora de diseñar planes de emergencia y protocolos de evacuación (Austin, 2013). Por otro lado, una mayor concentración de animales en lugares cerrados, ha sido valorada en la literatura, como un condicionante que incrementa su vulnerabilidad ante un posible riesgo (Bankoff, 2007). Un mayor número de animales residentes en un refugio, implica retos proporcionales en cuestión de logística, manipulación y transporte, especialmente en situaciones en las que el estrés de la situación, puede afectar a la conducta de los animales, habitualmente faltos de sociabilización, favoreciendo su agresividad con otros animales o con sus manipuladores, así como exacerbar problemas médicos (Dieckmann et al., 2020) 
Además, debemos contemplar la vulnerabilidad espacial de este tipo de centros por su localización, pues con frecuencia son construidos sobre suelos rústicos, de escaso valor comercial, y en algunos casos de difícil acceso. Este perfil responde a la necesidad de un buen número de metros cuadrados para la realización de sus actividades, cuyo valor de mercado resultaría muy superior en caso de ser suelos susceptibles de lucro o enriquecimiento. La relación entre capacidad económica y vulnerabilidad ha sido expuesta en diferentes trabajos, reflejando una tendencia a la ocupación de áreas de riesgo, por parte de las personas con menor poder adquisitivo (Daniel et al., 2009). En otros casos, son las entidades las que buscan lugares alejados y poco accesibles por motivos de seguridad para los animales residentes en sus instalaciones. Este tipo de ubicaciones marginales han demostrado ser notablemente más vulnerables ante los riesgos (Fernández \& D’Aragón, 2013).

A estos factores se añade el requisito de mantener una distancia mínima a los núcleos urbanos, establecido por varias comunidades y que es coherente con la reserva que muchos ayuntamientos hacen para conceder licencias de núcleo zoológico en lugares alejados, que pretenden evitar la difusión de enfermedades, así como reclamaciones de la población por ruidos, olores...2. En el momento actual, existe, en España, un borrador para la unificación de criterios, de nivel estatal, para constituir un núcleo zoológico ${ }^{3}$, que, si bien sigue manteniendo la necesidad de unas distancias mínimas que condicionan la ubicación de estos centros, también establecen la obligatoriedad de unos requisitos mínimos en sus infraestructuras internas.

La escasez de recursos económicos, la falta de planificación o la temporalidad de las instalaciones, condicionada por la inmediatez con la que estas organizaciones se ven obligadas a trabajar, han contribuido al incremento de la vulnerabilidad de los animales albergados. Esta vulnerabilidad económica, está marcada por el circulo vicioso entre la pobreza de infraestructuras y la capacidad para afrontar desastres. Este vínculo ha sido valorado, como uno de los factores más importantes de cara al proceso de recuperación tras un desastre (Hallegate et al., 2020). En el ejemplo etnográfico de AiBA Madrid, se observa cómo la infraestructura del Centro de Protección Animal, construido con fondos públicos resistió la nevada sin desperfectos y cómo fue la propia empresa contratada para la gestión de este centro (FCC), la que movilizó maquinaria pesada propia para despejar los accesos.

Por otra parte, se ha mostrado que la falta de herramientas legislativas, así como de protocolos específicos de actuación, es también clave en el aumento de la vulnerabilidad de los animales "de compañía" en caso de emergencia y desastre (White, 2012; Glassey \& Wilson, 2011). No existe PETS Act para los animales residentes en refugios españoles. En nuestro caso, la Ley 17/2015, de 9 de julio ${ }^{4}$, del Sistema Nacional de Protección Civil, asegura la coordinación de las políticas públicas de protección civil ante una emergencia o desastre, estableciendo un marco de protección jurídico. La redacción de esta ley, solo contempla a los humanos y sus bienes, pero no hace mención alguna de los demás animales. Esta ley nacional se desarrolla simultáneamente a las autonómicas. En Madrid, el Plan Territorial de Protección Civil de la Comunidad de Madrid (PLATERCAM) $^{5}$, establece la organización de respuesta ante situaciones de emergencia y desastre. Este plan tampoco tiene en cuenta a los animales no humanos en ningún apartado de su texto. Por el contrario, su análoga para Castilla la Mancha, PLATECAM ${ }^{6}$, vigente desde 2005, recomienda a la ciudadanía "conducir a los animales domésticos a lugares altos y protegidos", en caso de inundaciones, pero no establece obligaciones ni protocolos por parte de la administración pública.

Aunque en España, como en muchos otros países, los animales "de compañía" han sido históricamente considerados jurídicamente como "bienes", la falta de mención de éstos en las herramientas legislativas y protocolos de emergencia y desastres, hace que pasen desapercibidos. La ineptitud de esta consideración de los no humanos como bienes, así como su incapacidad para buscar soluciones coherentes, ha quedado repetidamente expuesta (Hernández, 2017). En el caso que nos ocupa, la falta de una estandarización y coordinación de los planes de emergencia y evacuación, deja a los animales "de compañía" a expensas de la decisión personal del responsable de una determinada operación, pasando de un plano legal u operacional a otro moral. Esta ausencia de criterio estandarizado contribuye a aumentar la vulnerabilidad de los animales no humanos en general y de los albergados en refugios en particular (Dieckmann et al., 2020).

En EEUU, la aplicación del modelo ICS por parte de la FEMA, así como la integración en él, de diferentes equipos profesionales para la atención sanitaria y rescate de los animales (CART,
2. Como ejemplo, puede consultarse el siguiente documento: DECRETO $181 / 2009$, de 20 de octubre, del Gobierno de Aragón, por el que se regulan los núcleos zoológicos en la Comunidad Autónoma de Aragón. Anexo II.

3. El borrador de Proyecto de Real Decreto por el que se establecen normas básicas de ordenación de los núcleos zoológicos, puede consultarse en la web del Ministerio español de Agricultura, pesca y Alimentación: https://www.mapa.gob.es/es/ganaderia/ participacion-publica/proyectordordenacionnucleoszoologicos_tcm $30-$ 542362.pdf

4. Ley 17/2015, de 9 de julio, del Sistema Nacional de Protección Civil. Jefatura del Estado «BOE» núm. 164, de 10 de julio de 2015. Referencia: BOE-A-2015-7730.

5. ACUERDO de 30 de abril de 2019 del Consejo de Gobierno, por el que se aprueba el Plan Territorial de Protección Civil de la Comunidad de Madrid. B.O.C.M. Núm. 113. Martes 14 de mayo de 2019. Pág. 83-238.

6. Decreto 36/2013, de 4 de julio, se regula la planificación de emergencias en Castilla-La Mancha y se aprueba la revisión del Plan Territorial de Emergencia de Castilla-La Mancha. D.O.C.M. núm. 129 de 05/o7/2013. 
SART, DART, VMAT), han demostrado ser eficaces para minimizar los efectos de un desastre, en lo que a los animales no humanos implica (Heath \& Linnabary, 2015). En España comunidades como Extremadura ${ }^{7}$ y Cantabria $^{8}$ reconocen la inclusión específica de las personas, animales y bienes en la gestión y coordinación de emergencias. Sin embargo, la mayoría de comunidades no tienen protocolos que incorporen a los animales no humanos en sus planes de emergencia y respuesta. Como excepción, cabe decir que la Comunidad Autónoma de Madrid, a través del Plan Especial de Protección Civil ante el Riesgo de Inundaciones (INUCAM) 9 , de 9 de diciembre de 2020, si menciona expresamente el rescate de animales domésticos en este tipo de situaciones, siempre y cuando hayan finalizado las labores de salvamento y rescate de las personas afectadas. Por su parte, la Unidad Militar de Emergencias, con competencias en este ámbito, no incorpora en su protocolo de intervención, referencia alguna a los animales ${ }^{10}$. En referencia a la crisis sufrida tras el paso de la borrasca Filomena, más allá de actuaciones particulares de gobiernos locales, el único pronunciamiento sobre la consideración de los animales no humanos de nivel estatal, se produjo el 13 de enero a través de la página de Facebook de la DGDDAA, en la que se recomendaba a las diferentes autoridades con competencias en la gestión de emergencias y desastres, que activasen mecanismos que tuviesen en cuenta las necesidades de los animales, con especial atención al acceso a los centros, así como a la alimentación y cuidado de colonias felinas urbanas.

Por otro lado, una de las mayores brechas puestas de manifiesto en el trabajo de Irvine (2007), tuvo que ver con la falta de formación y entrenamiento del voluntariado. La autora establece cómo, el manejo de voluntarios bien intencionados, pero sin formación, a los que se refiere como Spontaneous Untrained (or Unsolicited) Volunteers, puede constituir uno de los mayores retos para este tipo de organizaciones sin ánimo de lucro, que se soportan gracias a las ayudas particulares. Además, en diferentes estudios, se ha contemplado como un problema añadido, la gestión del riesgo que implica para estos primeros respondedores, cuya vulnerabilidad también se ve aumentada (Irvine, 2009:38; Mattes, 2018). En el trabajo de Decker et al. (2010), situado en EEUU, ampliamente familiarizado con los eventos climatológicos extremos, solo el $13 \%$ de los centros encuestados reconocieron tener planes de emergencia y protocolos por escrito, mientras que solo el $12 \%$ ha llevado a cabo algún entrenamiento al respecto. En España no existe ningún organismo público o privado que ofrezca formación específica sobre gestión de emergencias y desastres al voluntariado de los refugios. Se evidencia, por tanto, una deficiencia en la formación y entrenamiento del voluntariado español.

Por último, como ha quedado manifiesto a través de los ejemplos, la pertenencia a redes de relaciones públicas y privadas, así como la percepción ciudadana sobre la entidad o sus responsables juegan también un papel esencial en la vulnerabilidad de sus animales. Las teorías sobre el capital social en situaciones de emergencia y desastre, han sido contempladas por su importancia a la hora de reducir o aumentar la vulnerabilidad de determinadas comunidades, así como de influir en los procesos de recuperación (Panday et al., 2021; Durant, 2011; Malherbe et al., 2020). En el caso de Brinzal y Cuencanimal, el mantenimiento de estas relaciones y de una reputación social favorable, contribuyó a la rápida colaboración institucional y consiguiente disminución de la vulnerabilidad de sus animales.

\section{CONCLUSIONES}

La experiencia española en gestión de emergencias y desastres en lo que se refiere a animales no humanos, es bastante reducida. Sin embargo, la eventualidad de un desastre de origen antrópico local o a gran escala, puede acontecer en América Latina y el Caribe, y en cualquier otro lugar del mundo. Una vez sabemos que los desastres no son "naturales" y que nuestras actividades en el planeta tienen un impacto también sobre los demás animales, surge la responsabilidad de considerar, en lo que a la gestión de emergencias y desastres se refiere, a todos los animales en general, y a los que dependen por completo de nosotros (animales "de compañia"), en particular.

La experiencia de EEUU, especialmente a partir del paso del huracán Katrina, en 2005 y los más de 200.000 vidas de animales "de compañía" que se dejó atrás, debe hacernos reflexionar sobre la necesidad de estar preparados. Paradójicamente, los animales "de compañía" que residen en refugios y centros de recuperación, no han recibido apenas atención por parte de la comunidad científica, siendo uno de los colectivos más expuestos, por su total dependencia de los humanos. En concreto los animales residentes en este tipo de centros, en España, podrían estar expuestos a una alta vulnerabilidad en caso de emergencia y desastre. A través de las viñetas etnográficas

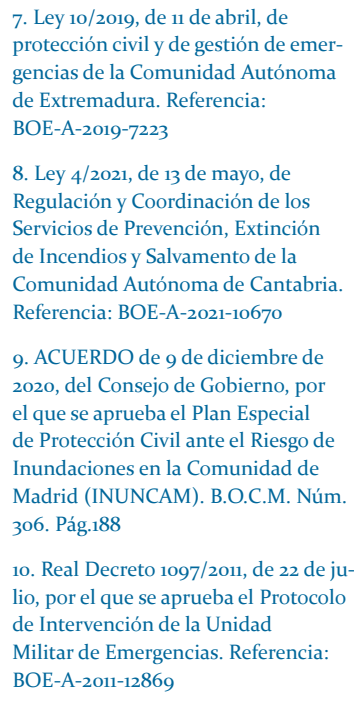


enmarcadas en el paso de la borrasca Filomena por la península Ibérica, a principios de 2021, este artículo, pone el foco sobre estos centros, mostrando la necesidad de establecer una estrategia integral para el manejo de emergencias y desastres, que no deje a ninguno atrás.

Gracias al esfuerzo personal de muchos profesionales y voluntarios y, sobre todo voluntarias, Filomena no se saldó con un número elevado de muertes en los refugios españoles, aunque sí con cuantiosas pérdidas económicas, que condicionan su capacidad de recuperación ante futuros desastres. Sin embargo, esta situación debe hacernos avanzar en varios sentidos. En primer lugar, desde las administraciones públicas, en la elaboración de herramientas legislativas de carácter estatal y autonómico, que contemplen las diferentes vulnerabilidades a las que se exponen los animales de los refugios. Estos instrumentos deben establecer regulaciones, protocolos y planes que permitan que las actuaciones locales se guíen por procedimientos estandarizados para garantizar la seguridad de los animales refugiados. En este contexto, la DGDDAA, puede y debe jugar un papel esencial. En segundo lugar, desde las organizaciones, estableciendo redes de colaboración, desarrollando planes de formación para sus profesionales y voluntarios, e implementando métodos de prevención y protocolos para la gestión de emergencias y desastres.

A partir de un estudio de caso concreto, localizado en España, este trabajo trata sobre la vulnerabilidad ante una emergencia 0 desastre, a la que están sometidos los animales de compañía que residen en refugios y protectoras, bien en periodo de recuperación, bien a la espera de adopción o acogimiento, o con carácter indefinido. Así pues, pretende contribuir a su incorporación en la gestión de emergencias y desastres de cualquier índole, siendo aplicable a todas las regiones del planeta, con especial foco en Latino América, Caribe y España. Incluir a los animales no humanos en la narrativa humanitaria de los desastres, nos ayuda a situarlos en el centro de una política en la que las relaciones entre humanos y demás animales, se articulan con otros sistemas para visibilizar las vulnerabilidades a las que estos últimos están sometidos. El despliegue de tecnologías humanitarias en este ámbito, no solo es importante para minimizar las vulnerabilidades a las que los no humanos se ven expuestos, sino que contribuye, de manera performativa, al desarrollo de un discurso humanitario que no discrimine a nadie. De este modo se defiende la idea de que, por duros que sean los fenómenos sufridos, tenemos una responsabilidad hacia los no humanos. Filomena también es nombre de animal.

\section{AGRADECIMIENTOS}

Los autores queremos agradecer a las personas colaboradoras de este trabajo, Puri, Lourdes, Patricia y Laura, así como a las instituciones que representan, la atención recibida y el tiempo dedicado durante las entrevistas y visitas que se realizaron a sus centros. La comprensión que han mostrado hacia esta investigación, es especialmente valorable, en un contexto de voluntariado en el que todos los esfuerzos se deben centrar en la atención a los animales. Sirve este agradecimiento, también como reconocimiento por su labor diaria. En particular, nos gustaría reconocer el incansable trabajo de la recientemente fallecida, Mercedes Culebras, fundadora y presidenta de Cuencanimal, quién dedicó gran parte de su vida a la defensa de los más vulnerables. Con este trabajo, pretendemos contribuir a la consideración y visibilización de los animales no humanos, en situaciones de emergencia y desastre.

\section{REFERENCIAS}

AEMET. (2021). Borrasca Filomena. AEMET. Disponible en: http://www.aemet.es/es/conocermas/ borrascas/2020-2021/estudios_e_impactos/filomena

Alfonso, P., \& Percedo, M.I. (2020). Estudio de caso. sector de la sanidad animal frente al huracán «Irma» en cuba. Revue Scientifique Et Technique (International Office of Epizootics), 39(2), 417-425.

Arluke, A. \& Sanders, C.R. (1996). Regarding Animals. Philadelphia: Temple University Press.

Austin, J.J. (2013). Shelter from the storm: Companion animal emergency planning in nine states. Journal of Sociology \& Social Welfare, 40, 185-210.

Bankoff, G. (2004). Time is of the essence: disasters, vulnerability and history. International Journal of Mass Emergencies and Disasters, 22(3), 23-42.

Bankoff, G. (2007). Bodies on the beach: domesticates and disasters in the Spanish Philippines 17501898. Environment and History, 13(3), 285-306.

Bankoff, G. (2014). Learning about disasters from animals. In Egner, H., Schorch, M., \& Voss, M. (Eds.), Learning and Calamities: Practices, Interpretations, Patterns (pp. 62-75). Routledge. 
Barnett, M. (2011). Empire of humanity: A history of humanitarianism. Cornell University Press.

Buttke, D., Vagi, S., Bayleyegn, T., Schnall, A., Morrison, M., Allen, M., \& Wolkin, A. (2013). Communication, information seeking, and evacuation plans for a disaster using Community Assessment for Public Health Emergency Response in the GulF Coast counties of Alabama and Mississippi, 2011. Journal of emergency management (Weston, Mass.), 11(3), 213-223.

Daniel, V.E., Florax, R.J., \& Rietveld, P. (2009). Flooding risk and housing values: An economic assessment of environmental hazard. Ecological Economics, 69(2), 355-365.

Decker, S.M., Lord, L.K., Walker, W.L., \& Wittum, T.E. (2010). Emergency and disaster planning at Ohio animal shelters. Journal of Applied Animal Welfare Science, 13(1), 66-76.

Dennison, K. M., \& Lin, J. (2012). Disaster and Emergency Planning and Response for Animal Shelters. In Miller, L., \& Zawistowski, S. (Eds.), Shelter medicine for veterinarians and staff(517-528). John Wiley \& Sons.

Díaz, A., Trelles, S., \& Murillo, J.C. (2015). La gestión del riesgo y la atención de animales en desastres. Instituto Interamericano de Cooperación para la Agricultura (IICA), Costa Rica.

Dieckmann, H.G., Costa, L.R., \& Madigan, J.E. (2020). Current operational model for veterinary care in large animal shelters during disasters. Prehospital and disaster medicine, 35(5), 579-587.

Douglas, R., Kocatepe, A., Barrett, A.E., Ozguven, E.E., \& Gumber, C. (2019). Evacuating people and their pets: older Floridians' need for and proximity to pet-friendly shelters. The Journals of Gerontology: Series B, 74(6), 1032-1040.

Durant, T.J. (2011). The Utility of Vulnerability and Social Capital Theories in Studying the Impact of Hurricane Katrina on the Elderly. Journal of Family Issues, 32(10), 1285-1302.

Farmer, A.K., DeYoung, S.E., \& Wachtendorf, T. (2016). Pets and evacuation: an ongoing challenge in disasters. Journal of homeland security and emergency management, 13(4), 1-13.

Farmer, A.K., \& DeYoung, S.E. (2019). The pets of Hurricane Matthew: Evacuation and sheltering with companion animals. Anthrozoös, 32(3), 419-433.

Fatjó, J., \& Calvo, P. (2018). Affinity Foundation Study into the abandonment, loss and adoption of pets in Spain. Affinity Foundation

Fernández, R.A.F., \& D’Aragon, J. (2013). Understanding slums' vulnerability to disaster risks through their spatial configuration. Regional Development Dialogue, 34(1), 63-82.

Gimenez, T., Gimenez, R.M., Baker, J.L., \& Johannessen, D.T. (2002). How to effectively perform emergency rescue of equines. Am. Assoc. Eq. Pract, 48, 276.

Glassey, S., \& Wilson, T.M. (2011). Animal welfare impact following the 4 September 2011 Canterbury (Darfield) earthquake. Australasian Journal of Disaster and Trauma Studies, 2, 49-59.

Hallegatte, S., Vogt-Schilb, A., Rozenberg, J., Bangalore, M., \& Beaudet, C. (2020). From poverty to disaster and back: A review of the literature. Economics of Disasters and Climate Change, 4(1), 223-247.

Heath, S.E., Voeks, S.K., and Glickman, L.T. (2001). Epidemiological Features of Pet Evacuation Failure in a Rapid-Onset Disaster. Journal of the American Veterinary Medical Association, 218, 1898-1904.

Heath, S.E., \& Linnabary, R.D. (2015). Challenges of managing animals in disasters in the US. Animals, $5(2), 173-192$.

Hernández, J.G. (2017). El animal de compañía como objeto jurídico especial: Su estudio específico en la comunidad de bienes. Revista CESCO de Derecho de Consumo, (21), 50-89.

Hernández-Pulido, D., Carrillo, R.F., Hernández-Gallo, N., Bernal, L.A., \& Jiménez-Alonso, G. (2021). Animales en la gestión del riesgo de los desastres. Avances, perspectivas y casos de estudio. En Comisión Nacional Asesora para la Investigación en Gestión del Riesgo de Desastres (Eds.), Investigaciones en gestión del riesgo de desastres para Colombia: avances, perspectivas y casos de estudio (pp.174-191). Unidad Nacional para la Gestión del Riesgo de Desastres (UNGRD).

Howlett, M., \& Turnbull, C. (2009). Large animal emergency rescue training. Australia, Agrifoods Skills Australia International Specialised Skills Institute.

Hunt, M.G., Bogue, K., \& Rohrbaugh, N. (2012). Pet ownership and evacuation prior to Hurricane Irene. Animals, 2(4), 529-539.

Irvine, L. (2004). Providing for pets during disasters: An exploratory study. Department of Sociology University of Colorado. 
Irvine, L. (2007). Ready or not: Evacuating an animal shelter during a mock emergency. Anthrozoös, 20(4), 355-364.

Irvine, L. (2009). Filling the Ark. Temple University Press.

Madigan, J., \& Dacre, I. (2009). Preparing for veterinary emergencies: disaster management and the Incident Command System. Revue scientifique et technique (International Office of Epizootics), $28(2), 627-633$.

Malherbe, W., Sauer, W., \& Aswani, S. (2020). Social capital reduces vulnerability in rural coastal communities of Solomon Islands. Ocean E Coastal Management, 191, 105186.

Mattes, S. M. (2018). Animals left behind: Multispecies vulnerability in post-3-11 Japan. Michigan State University.

Morales, M.I., Jiménez, I., \& López, M.B. (2017). Los damnificados invisibles. 167-185. En Costa, M. (Ed.), Intervenciones psicosociales en emergencias y desastres Construcciones desde la experiencia. Editorial Brujas.

Oliver-Smith, A. (2009). Understanding Hurricane Mitch: Complexity, Causality, and the Political Ecology of Disasters. En Ensor (Ed.), The Legacy of Mitch: Lessons from Post-Disaster Reconstruction in Honduras. The University of Arizona Press.

Panday, S., Rushton, S., Karki, J., Balen, J., \& Barnes, A. (2021). The role of social capital in disaster resilience in remote communities after the 2015 Nepal earthquake. International Journal of Disaster Risk Reduction, 55, 102112.

Petrolia, D.R., \& Bhattacharjee, S. (2010). Why don't coastal residents choose to evacuate for hurricanes? Coastal Management, 38(2), 97-112.

Ticktin, M.I. (2014). Humanitarianism as Planetary Politics. En Perera, S. \& Razack, H. (Eds.), At the Limits of Justice: Women of Colour on Terror (pp.406-419). University of Toronto Press

Ticktin, M. (2015). Non-human suffering: A humanitarian project. In I. Harper, T. Kelly, \& A. Khanna (Eds.), The Clinic and the Court: Law, Medicine and Anthropology (pp. 49-71). Cambridge University Press.

Tobias, M.C., \& Morrison, J.G. (2017). Anthrozoology. Embracing Co-Existence in the Anthropocene. Springer. Los Angeles, USA

Travers, C., Degeling, C., \& Rock, M. (2017). Companion animals in natural disasters: A scoping review of scholarly sources. Journal of Applied Animal Welfare Science, 20(4), 324-343.

Vieira, A.D.P., \& Anthony, R. (2021). Reimagining Human Responsibility Towards Animals for Disaster Management in the Anthropocene. In Bovenkerk \& J. Keulartz (Eds.), Animals in Our Midst: The Challenges of Co-existing with Animals in the Anthropocene (pp.223-254). Springer.

White, S. (2012). Companion animals, natural disasters and the law: An Australian perspective. Animals, 2, 380-394.

Yglesias, M.S.E.M. (2015). Los animales y la gestión del riesgo de desastre. Revista en Torno a la Prevención, (14), 22-29.

Zottarelli, L.K. (2010). Broken bond: An exploration of human factors associated with companion animal loss during Hurricane Katrina. Sociological Forum, 25(1), 110-122. 